Rev. Elev. Méd. Vét. Pays, trop., 1967, 20, I (115-119)

\title{
Teneur du muscle en glycogène et identification des viandes falsifiées
}

\author{
par Shokry H. KAMEL, Tahony M. IBRAHIM, Ahlami A. AFIFI \\ Service de médecine légale, Faculté de médecıne vétérinaire, Giza, République Arabe Unie
}

\section{RÉSUMÉ}

1. - Une épreuve chimique permettant de déterminer la teneur tatale en glycogène des muscles de différents animaux domestiques est décrite.

La différenciation dépend de la variation de l'intensité de 'a couleur mesurée au colorimètre.

2. - L'épreuve peut être utilisée pour la diagnose de la viande foetale (non comestible) et de toutes les cutres varıétés de viande.

3. - Elle peut également permettre de difiérencier très bien !a viande de buffle de celle du bceuf, et cette dernière de celle du chameau. La différenciation entre la viande de chameau et de buffle devra être confirmée par d'autres méthodes.

4. - Les résultats obtenus avec la viande de mouton et celle de chèvre sont presque semblables et se superposent à ceux obtenus avec la viande de veau mais heureusement dans chaque cas les caractéristiques naturelles peuvent aider à faire la distinction.

5. - L'analyse statistique des valeurs obtenues à la suite des mesures effectuées sur différentes régions du corps et chez différents animaux est enregistrée.

\section{INTRODUCTION}

L'un des problèmes primordiaux qui existent dans notre vie professionnelle quotidienne est la question des viandes falsifiées. Les experts de médecine légale ont souvent à se prononcer sur de tels cas. Ces jugements destinés à distinguer différentes sortes de viandes apparemment identiques sont la plupart du temps basés sur l'examen macroscopique. Cette ressemblance pourrait en effet être utilisée par certaines personnes pour substituer des viandes de qualités inférieures à des carcasses de bonne qualité. Les exemples rencontrés le plus communément concernent la substifution de viande de chameau et de cheval à celle de bouf ou de buffle,de viande de chèvre à celle de mouton et de viande fotale à celle de veau. Bien que la différenciation macroscopique puisse être corroborée sérolo- giquement par l'épreuve des précipitines, la nécessité d'une épreuve chimique a donné l'idée d'essayer cette identification en mesurant par colorimétrie les quantités variables de glycogène présent dans tous les tissus de toutes les espèces animales. Dans ce but des animaux de différentes espèces apparemment en bonne santé et dans des conditions reconnues normales ont été soumis à l'épreuve. Les muscles ont été choisis en raison de leur taux de glycogéne réputé plus stable que celui du foie car dans celui-ci la quantité de glycogène varie beaucoup en fonction de plusieurs facteurs tels que : âge, nutrition, fatigue et maladie.

\section{MATÉRIEL ET MÉTHODES}

Des échantillons de viande ont été prélevés sur des animaux apparemment en bonne santé 
après leur abattage. Pour éviter les effets de la fatıgue, de la maladıe et des variations alimentaires sur le taux du glycogène musculaire, on $n$ 'a utilisé pour les expériences que des animaux précédemment soumis à une quarantaine (ELAFIFI et ELMOSSALAMI, 1960-1961).

En quarantaine les anımaux sont en général privés de toute nourriture pendant 24 heures avant l'abattage mais boivent de l'eau à volonté. On a utilisé pour cette expérience des buffles et des chameaux de plus de 5 ans, des bovins âgés de 1 à 3 ans, des moutons et des chèvres de plus de 2 ans ainsi que des veaux et des fætus; on a prélevé des échantillons de viande sans graisse sur 20 animaux de ces différentes espèces. Un prélèvement des muscles de la tête, de l'encolure, de l'épaule, de l'abdomen et de la cuisse a été effectué sur chacun de ces animaux.

Les échantillons épongés pour enlever le sang adhérant ont été gardés au réfrigérateur durant 2 heures après l'abattage. Chaque échantıllon a été ensuite soumis à l'épreuve afin de déterminer son taux de glycogène.

La technique adoptée pour cet essai était celle décrite par NERURKAR et SAHASRBUDLE (1959), dont les 3 phases sont décrites ci-dessous.

Le but de la première phase de l'épreuve est de retirer des tissus le glucose libre ef les glucoses phosphates (POLTER et ELVEHJEM 1936).

La seconde phase consiste à extraire par la chaleur le glycogène du résidu obtenu de la première phase (KEMP et KITS Van HEIJNINGEN 1954).

Dans la troisıème phase ou hydrolyse le glycogène des tissus en glucose afin d'exprimer la teneur en glycogène par son équivalent en glucose (MENDAL et Coll., 1954).

Le glucose est alors dosé par la réaction de Dische (GRUIN ef HOOD, 1939), modifiée par NILSSON (1958).

Pour extraire le glycogène, un demi-gramme d'un échantillon muscularre haché estbien homogénéisé avec du méthanol froid à 80 p. 100 dans un verre. Le produit homogénéisé est ensuite centrifugé à 3.000 tours/minute pendant 15 minutes et le liquide surnageant contenant le glucose libre et le glucose phosphate (POLTER ef ELVEHJEM, 1936) est éliminé, Le résidu est ensuite lavé plusieurs fois avec du méthanol à 80 p. 100 pour assurer l'élimınatıon complète des dernières traces de glucose. Le résidu est ensuite chauffé avec $8 \mathrm{ml}$ d'acide trichloracétique à 5 p. 100 à $100^{\circ} \mathrm{C}$ pendant 15 minutes afin d'extraire la totalité de son glycogène et centrifugé à 3.000 tours/minute pendant encore 15 minutes (KEMP et KITS Van HEIJNINGEN, 1954). La solution claire surnageante contient la totalité du glycogène. Pour déterminer la quantité de glycogène on ajoute $1,8 \mathrm{ml}$ de la solution d'acide trichloracétique à $5 \mathrm{p}$. 100 à un échantillon de $0,2 \mathrm{ml}$ de la précédente solution claire surnageante dans un grand tube à essai et l'on refroidit le tout dans un mélange réfrigérant glace et sel.

Puis on ajoute au contenu du tube $9 \mathrm{ml}$ d'une solution d'acide sulfurique préparée à l'avance par dilution de 8 parties d'acide sulfurique concentré de poids spécifique 1,84 avec une partie d'eau distillée. On secoue le tube ef on laisse refroidir. On ajoute ensulte à chaque tube à essai $0,3 \mathrm{ml}$ d'une solution à $0,5 \mathrm{p} .100$ de carbazole dans l'alcool éthylique. Les tubes à essai sont ensuite chauffés au bain-marie à $100^{\circ} \mathrm{C}$ pendant 15 minutes jusqu'à ce qu'une teinte rose se manifeste. On refroidit de nouveau les tubes à essai sous l'eau du robinet et on les transvase dans les tubes propres et secs du colorimètre: l'intensité de la teinte qui est stable est mesurée avec un colorimètre lumetron à longueur d'onde de $550 \mathrm{~m} \mu$ après avoir étalonné l'appareil à la concentration 0 avec de l'eau distillée.

Pour pouvoir tracer une courbe étalon on prépare différentes dilutions standard d'une quantité connue de glucose dans une solution d'acide trichloracétique à 5 p. 100 . On utilise pour chaque échantillon la technique précédemment décrite. La densité optique pour ces dilutions standards traitées est mesurée à $550 \mathrm{~m} \mu$ par rapport à la concentration 0 . La courbe étalon est ensuite tracée en notant les concentrations de glucose correspondantes cux lectures de densité optique.

L'analyse statistique des résultats obtenus a été faite pour mesurer les différences de taux de glycogène entre chacune des 2 espèces d'animaux concernées dans la falsification.

La méthode d'analyse de variance «Split-plot 》 (SNE-DECOR, 1961) a été adoptée pour mesurer les différences de teneur en glycogène entre les espèces, dans les différentes parties du corps ainsi que les interactions entre les espèces ef les parties du corps. 


\section{RÉSULTATS}

La teneur totale en glycogène estimée d'après l'analyse chimique des différents échantillons de viande collectés dans différentes parties du corps chez plusieurs espèces animales est enregistrée dans le tableau $n^{\circ} \mid$ ainsi que le calcul de leurs déviations standards.

Le tableau fait apparaître des valeurs différentes selon les régıons du corps d'un même anı$\mathrm{mal}$ et, pour des régions correspondantes, selon les espèces.

Ces différences de valeurs ont été analysées statistiquement pour apprécier leur signification. Les résultats de cefte analyse sont résumés dans le tableau no 2 .

\section{DISCUSSION}

A partir des résultats obtenus, on peut voir que la plus forte concentration de la teneur en glycogène se trouve dans les muscles de la cuisse de tous les animaux. La concentration la plus basse varie avec chaque espèce :

Chez le buffle ce sont les muscles de l'encolure, chez le chameau, les muscles de la paroi abdominale, ainsi que chez le mouton, la chèvre ef le fœtus, tandis que chez le bauf et le veau ce sont les muscles de la tête.

En ce qui concerne la viande de buffle ef de chameau, il existe une grande similitude de leur teneur en glycogène, outre la présence de données qui se recouvrent entre elles pour les muscles de la cuisse du buffle $5.38 \pm 0.719$ et pour ceux du chameau $6.74 \pm 0.79$. Ces résultats conduisent à la limitation de l'emploi du test chez ces espèces.

Néanmoins l'apparence naturelle, concernant la couleur de la viande, le caractère du muscle et le taux de graisse allant de pair avec la consistance des fibres musculaires, fournissent une aide appréciable pour différencier le buffle ef le chameau. D'ailleurs il y a une variation frappante entre le bœuf d'une part $(2,81 \pm 0,706$ dans le muscle de la cuisse) et le chameau et le buffle d'autre part. Ainsi dans ce cas, on peut davantage se fier à cette épreuve car il n'y a qu'une légère concordance dans toutes les parties, sauf pour les muscles de l'abdomen.

Aucune différence n'a pu également être tróuvée dans la teneur en glycogène des muscles du mouton et de la chèvre. Néanmoins on $y$ : constate des différences significatives selon les régions. L'échantillon prélevé dans la cuisse' montre la plus haute teneur en glycogène: " :

$2,07 \pm 0,229$ chez le mouton;

$2 \pm 0,286$ chez la chèvre.

II existe une légère différence entre le glyco-: gène musculaire de ces espèces et celui de la: viande de veau.

Une différence frappante existe entre la teneur en glycogène des muscles de ces petits animaux comestibles et celle de la viande foetale, ce quí montre que l'épreuve pourrait être tout à fait valable. Dans ce cas particulier les auteurs estiment que la différence trouvée entre le vecu et le fœius pourrait être attribuée aux différences de teneur en eau laquelle est probablement plus facile à déterminer.

En conclusion, l'estimation de la teneur du muscle en glycogène comme moyen d'identification des falsifications de la viande est fidèle pour certaines espèces tandis qu'elle ne l'est pas complètement pour d'autres chez lesquelles les caractères naturels de la viande doivent être pris en considération.

\section{REMERCIEMENTS}

Les duteurs tiennent à remercier le Docteur A. H. SAID pour ses conseils et son aide précieuse. 
TAkiLAU NO I

Montrant les valeurs moyennes, les écarts-types et les valeurs extrêmes de la teneur totale en glycogène dans les différentes parties du corps de diverses espèces animales.

\begin{tabular}{|c|c|c|c|c|c|c|}
\hline \multirow{2}{*}{ Espèces } & \multirow{2}{*}{ Valeurs } & \multicolumn{5}{|c|}{ mg. de glycogène total par mg. de tissu } \\
\hline & & Tete & Fncolure & Epaules & Abdomen & Cuisse \\
\hline $\begin{array}{l}\text { Buffles de plus } \\
\text { ae } 5 \text { ans. }\end{array}$ & $\begin{array}{l}\text { moyenne } \\
\text { extrêmes }\end{array}$ & $\begin{array}{l}4,26 \pm 0,855 \\
2,6 \text { غे } 5,3\end{array}$ & $\begin{array}{l}3,22 \pm 0,504 \\
2,2 \text { दे } 5,1\end{array}$ & $\begin{array}{l}4,9 \pm 0,71 \\
3,9 \pm 6,0\end{array}$ & $\begin{array}{l}4,0 \pm 1,04 \\
2,7 \pm 5,6\end{array}$ & $\begin{array}{l}5,38 \pm 0,719 \\
4,5 \text { a } 6,8\end{array}$ \\
\hline $\begin{array}{l}\text { Boeufs de } \\
1 \text { à } 3 \text { ans. }\end{array}$ & $\begin{array}{l}\text { moyenne } \\
\text { extrêmes }\end{array}$ & $\begin{array}{l}1,68 \pm 0,437 \\
1,2 \text { à } 2,7\end{array}$ & $\begin{array}{l}1,96 \pm 0,473 \\
1,2 \approx 4,4\end{array}$ & $\begin{array}{l}2,06 \pm 0,513 \\
1,2 \text { घ } 4,0\end{array}$ & $\begin{array}{l}1,9 \pm 0,475 \\
1,2 \pm 2,6\end{array}$ & $\begin{array}{l}2,81 \pm 0,706 \\
1,2 \text { i } 4,8\end{array}$ \\
\hline Chameaux & $\begin{array}{l}\text { moyenne } \\
\text { extrêmes }\end{array}$ & $\begin{array}{l}5,6 \pm 1,02 \\
3,9 \text { à } 7,2\end{array}$ & $\begin{array}{l}4,28 \pm 0,861 \\
3,0 \text { à } 5,3\end{array}$ & $\begin{array}{l}5,54 \pm 1,12 \\
3,8 \text { à } 7,2\end{array}$ & $\begin{array}{l}4,12 \pm 0,759 \\
3,2 \text { à } 5,2\end{array}$ & $\begin{array}{l}6.74 \pm 0,79 \\
5.9 \text { غे } 8,7\end{array}$ \\
\hline Noutons & $\begin{array}{l}\text { moyenne } \\
\text { extrênes }\end{array}$ & $\begin{array}{l}1,52 \pm 0,268 \\
1,1 \text { à } 2,0\end{array}$ & $\begin{array}{l}1,57 \pm 0,366 \\
1,0 \text { à } 2,1\end{array}$ & $\begin{array}{l}1,6 \pm 0,272 \\
1,2 \text { à } 2,0\end{array}$ & $\begin{array}{l}1,46 \pm 0,332 \\
1,0 \text { ㄹ } 2,0\end{array}$ & $\begin{array}{l}2,07 \pm 0,229 \\
1,7 \text { в̀ } 2,5\end{array}$ \\
\hline Chèvres' & $\begin{array}{l}\text { moyenne } \\
\text { extrêmes }\end{array}$ & $\begin{array}{l}1,65 \pm 0,344 \\
1,1 \text { à } 2,1\end{array}$ & $\begin{array}{l}1,6 \pm 0,316 \\
1,3 \text { à } 2,1\end{array}$ & $\begin{array}{l}1,66 \pm 0,28 \\
1,2 \text { a } 2,0\end{array}$ & $\begin{array}{l}1,42 \pm 0,282 \\
1,1 \text { ̀े } 1,8\end{array}$ & $\begin{array}{l}2,0 \pm 0,286 \\
1,6 \text { ¿ } 2,4\end{array}$ \\
\hline Veaux & $\begin{array}{l}\text { moyenne } \\
\text { extrémes }\end{array}$ & $\begin{array}{l}1,18 \pm 0,24 \\
0,9 \text { à } 1,8\end{array}$ & $\begin{array}{l}1,24 \pm 0,303 \\
0,9 \text { à } 1,8\end{array}$ & $\begin{array}{l}1,32 \pm 0,296 \\
0,8 \text { à } 1,8\end{array}$ & $\begin{array}{l}1,28 \pm 0,218 \\
1,0 \text { ̀ } 1,8\end{array}$ & $\begin{array}{l}1,7 \pm 0,415 \\
1,0 \text { a } 2,6\end{array}$ \\
\hline Foetus & $\begin{array}{l}\text { moyenne } \\
\text { extrémes }\end{array}$ & $\begin{array}{l}0,3 \pm 0,08 \\
0,1 \text { ¿ } 0,5\end{array}$ & $\begin{array}{l}0,29 \pm 0,113 \\
0,1 \text { à } 0,5\end{array}$ & $\begin{array}{l}0,26 \pm 0,106 \\
0,1 \text { а̀ } 0,6\end{array}$ & $\begin{array}{l}0,24 \pm 0,06 \\
0,1 \text { à } 0,4\end{array}$ & $\begin{array}{l}0,32 \pm 0,10 \\
0,1 \text { à } 0,6\end{array}$ \\
\hline
\end{tabular}

TABLEAU NO II

Analyge de la varlance appliquée aux différento résultats obtenus

\begin{tabular}{|c|c|c|c|c|c|}
\hline \multirow[b]{2}{*}{ Sources de variatıons } & \multirow[b]{2}{*}{ Degré de liberté } & \multicolumn{4}{|c|}{ Carté moyen } \\
\hline & & $\begin{array}{c}\text { Buffle } \\
\text { Chameau (1) }\end{array}$ & $\begin{array}{c}\text { Bout } \\
\text { Buffle (2) }\end{array}$ & $\begin{array}{c}\text { Mouton } \\
\text { Chevres (3) }\end{array}$ & $\begin{array}{c}\text { Veau } \\
\text { Foetus (4) }\end{array}$ \\
\hline Espèce & 1 & $20,43 * *$ & 135,07 ** & 0,01 & $28,20 * *$ \\
\hline $\begin{array}{l}\text { Individus d'une même espèce } \\
\text { (erreur a) }\end{array}$ & 18 & 2,32 & 0.85 & 0,23 & 0,07 \\
\hline Régions & 4 & $17.15 * *$ & $6.83 * *$ & $1,00 * *$ & $0,25 *$ \\
\hline Espèce régrion & 4 & $1,39 *$ & $0.39 * *$ & 0,04 & $0,18 *$ \\
\hline Errewr (b) & 72 & 0,45 & 0,065 & 0,07 & 0,07 \\
\hline
\end{tabular}

(1) Analyse de variance entre le buffle de plus de 5 ans et le chemeau

(2) " " " le boeuf de 1 k̀ 3 ans et le buffle de plus de 5 ans

(3) " " le mouton et la chèrre

(4) " $" n$ le veau et le foetus

** Significatif au taux de $t$ p.t00 


\section{SUMMARY}

Muscle glycogen content as an aid for identification in meat adultration

1. - A chemical test for total glycogen content determination in muscles of different form animals is described. Differentiation depends on variation in intensity of colour developed as measured colorimetrıcally.

2. - The test is reliable for differentiation between faetus (non edible meat) and all other kinds of meat.

3. - The test could be carried out successfully in differentiating between buffaloe and cattle meat and between the latter and camel meat. Differentiation between comel and buffaloe should be aided by olher methods.

4. - Sheep and goat meat are nearly similar in results and overlapping with the data obtained from veal meat, but fortunately for every case natural characteristıcs can heip.

5. - Statistical analysis of values obtained from testing various parts of the body regions in different animals are recorded.

\section{RESUMEN}

La tasa del glicógeno del músculo como medio para identificar las adulteraciones concernientes a la origen de una carne

1. - Se describe una pruebo química permitiendo determinar la tasa total de glícógeno de los músculos en varios anımales domésticos. La díferenciación depiende de la varıación de la intensıdad del color medıda con el calorímetro.

2. - Se puede utilizar la dicha prueba para la diagnósis de la carne fetal (no comestible) y de todas las otras variedades de corne.

3. - Permite tambien diferenciar muy bien la carne de búfalo de la de vaca, y la última de la de camello.

Se necesitaran otras métodos para identificar la carne de camello de la de búfalo.

4. - Los resultados oblenidos con la carne de oveja y la de cabra son casi semejantes y se superponen con los obtenidos con la carne de ternera, pero en cada caso las características naturales pueden permitir la identificación.

5. - Se nota el análisis estadístico de los datos obtenıdos luego de medidas efectuadas sobre diferentes partes del cuerpo y en varios animales.

\section{BIBLIOGRAPHIE}

ELAFIFI (A.), ELMOSSALAMI (E.). - Vef. Med. J., Faculty of Vet. Med., Carro Univ., GizaEgypt, 1960-1961, 7 (7-8) : 324.

GRUIN (S.), HOOD (D. B.), - J. Biol. Chem. $1939,131: 211$.

$\operatorname{KEMP}$ (A.), KITS VAN HEIJNINGEN (A. J. M.). — Biochem. J., 1954, $56: 646$.

MENDEL (B.), KEMP (A.), MYERS (D. K.).Biochem. J., 1954, $56: 639$.
NERURKAR (M. K.), SAHASRABUDLE (M. B.). —Brif. J. exp. Path., 1959, 40 (4) : 318.

NILSSON (T.). - Acto. Path. Microbiol., Scand. Sup., 1958, 125, 29.

POLTER (V. R.), ELVEHJEM (C. A.). - J. Biol. Chem., 1936, 114, 495.

SNEDECOR (G.W.). - Statistical Methods, 5th Ed., lowa State Univ. Press, U. S. A., 1961. 